\title{
A Multi-Model Filter for Mobile Terminal Location Tracking
}

\author{
M. McGuire , K.N. Plataniotis \\ The Edward S. Rogers Sr. Department of Electrical and Computer Engineering, \\ University of Toronto, 10 King's College Road, Toronto, Ontario, M5S 3G4, email: mmcguire@dsp.toronto.edu
}

\begin{abstract}
Mobile terminal location is an important area of research because of its application in location-sensitive browsing and resource allocation. This paper presents a method for reducing the error in mobile terminal location estimation. A model-based dynamic filter is presented which uses an accurate model of mobile terminal motion to combine information from location measurements made at different time instances together to create an improved location estimate. The model of mobile terminal motion has a kinematic state space model describing the physical rules governing terminal motion and a control model that describes the human control input into the motion process. The dependency between the terminal location and the control decisions is used to derive a new dynamic filter. This filter provides better accuracy than previously known location techniques and is robust to variations in the mobile terminal motion.
\end{abstract}

\section{INTRODUCTION}

Much research has been performed on mobile terminal location in wireless cellular networks. Terminal location can be used for emergency communications and resource allocation assistance. For next generation networks, location methods based on Time of Arrival (ToA) and Time Difference of Arrival (TDoA) measurements are the subject of the most research since the proposed access and modulation schemes make accurate time measurements possible[1], [2]. No matter what the form of the measurements used to locate the mobile terminal, there are errors in the position estimates resulting from noise in the measurement data.

It has been shown that filtering of the location estimates can reduce the location errors[3]. The filtering algorithm improves the estimation of mobile terminal location by combining the information from measurements made at several sampling time periods together into an improved location estimate. A filter's error reduction performance is highly dependent on the relationship between the filter's structure and the properties of the random processes that compose the motion and measurement generation processes for the mobile terminals. For the filters presented in previous work, the relationship between the parameters of the filters and the real world attributes of mobile terminal motion were not well described.

This paper presents a location system with a filter based on an accurate model of mobile terminal motion. The mobile terminal

Partially supported by a grant from the Nortel Institute for Telecommunications motion model consists of a kinematic model, which describes the physical rules controlling the motion of mobile terminal motion, and a user control input decision model, which describes the user decisions concerning the motions of the mobile. The parameters of the models are easily computed from field measurements. The presented method is a network-based location solution since only the cellular network can have access to the necessary information about the local propagation and physical environment around the mobile terminal that is required by the location estimate filters.

Section II describes the measurement model. The radio propagation environment used to evaluate the location procedure is discussed and how the radio measurements are preprocessed before the filtering procedure is outlined. Section III describes the motion model for the mobile terminals. The kinematic and user control input models which dictate mobile terminal motion behavior are described. The model-based filter and control input estimator are described in Section IV. The results of simulations used to evaluate the filter are presented within Section V. Our conclusions are summarized in Section VI.

\section{Propagation and Measurement Model}

The measurements used to estimate mobile terminal location are propagation distance measurements based on ToA measurements. The entries of the propagation distance measurement vector, $\boldsymbol{Z}(k)$, are given by

$$
\boldsymbol{Z}_{j}(k)=\boldsymbol{d}_{j}(k)+\boldsymbol{\varepsilon}_{j}(k),
$$

where $j$ denotes the base station, $\boldsymbol{Z}_{j}(k)$ is the measurement of base station $j$ at sample interval $k, \boldsymbol{d}_{j}(k)$ is the propagation distance from base station $j$ to the mobile terminal at sample interval $k$ and $\varepsilon_{j}(k)$ is a random variable representing measurement noise for base station $j$. The measurement noise is Gaussian with a mean and a standard deviation, $\sigma_{d}$, of 16.0 meters. The parameters of the noise density are taken from [1], which simulated multipath propagation based on the COST 207 urban power delay profile. It is assumed that the measurement noise terms for different base stations are independent.

\section{A. Zero Memory Estimation}

For a given time interval $k$, the zero memory estimator calculates an estimate of the mobile terminal position, $\boldsymbol{Y}(k)$, from 


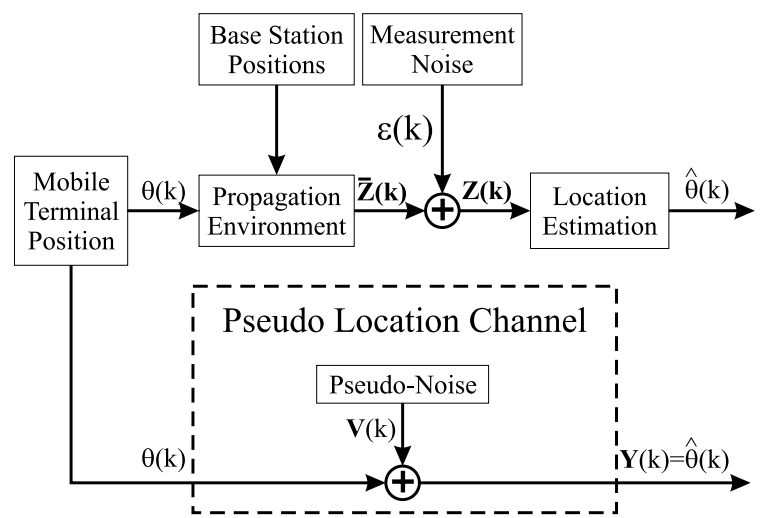

Fig. 1. Zero Memory Estimator for ToA location estimation

the measurement vector, $\boldsymbol{Z}(k)$. The so-called zero memory estimator is a preprocessor for the location estimation system. It allows the model-based location estimation filter to operate without knowledge of the non-linearities in the propagation environment. The conceptual model of the system is shown in Figure 1.

We propose the use of zero memory estimator which uses a survey of propagation measurements, $\left\{\boldsymbol{Z}_{1}, \boldsymbol{Z}_{2}, \ldots, \boldsymbol{Z}_{n}\right\}$ taken at known locations, $\left\{\boldsymbol{\theta}_{1}, \boldsymbol{\theta}_{2}, \ldots, \boldsymbol{\theta}_{n}\right\}$, in the network environment to estimate the mobile terminal's location from its measurement vector. This survey data can be obtained from field measurements or generated using computer ray-tracing propagation models. These ray-tracing models and survey measurements are often employed to predict and check network performance[4], [5].

The zero memory estimated location is calculated using [6]:

$$
\boldsymbol{Y}(k)=\hat{\boldsymbol{\theta}}(k)=\sum_{j=1}^{n} \boldsymbol{\theta}_{j} w_{j}(\boldsymbol{Z}(k))
$$

where

$$
w_{j}(\boldsymbol{Z}(k))=\frac{K_{z}\left(\frac{\boldsymbol{Z}(k)-\boldsymbol{Z}_{j}}{h_{z}}\right)}{\sum_{i=1}^{n} K_{z}\left(\frac{\boldsymbol{Z}(k)-\boldsymbol{Z}_{i}}{h_{z}}\right)},
$$

where $n$ is the number of survey points, $m$ is the length of the measurement vector $\boldsymbol{Z}(k), h_{z}=2 \sigma_{d}$ and

$$
K_{z}(\boldsymbol{Z})=(2 \pi)^{\frac{m}{2}} \exp \left(-\frac{\boldsymbol{Z}^{T} \boldsymbol{Z}}{2}\right) .
$$

An estimate of the covariance of the zero memory estimated location can be calculated using $\boldsymbol{R}(k)=\operatorname{Cov}(\boldsymbol{\theta}(k) \mid \boldsymbol{Z}(k))=$ $\mathrm{E}\left[\boldsymbol{\theta}(k) \boldsymbol{\theta}(k)^{T} \mid \boldsymbol{Z}(k)\right]-\mathrm{E}[\boldsymbol{\theta}(k) \mid \boldsymbol{Z}(k)] \mathrm{E}[\boldsymbol{\theta}(k) \mid \boldsymbol{Z}(k)]^{T}$. It has been shown that $\boldsymbol{Y}(k)$ from (2) calculates an estimate of $\mathrm{E}[\boldsymbol{\theta}(k) \mid \boldsymbol{Z}(k)]$ [6]. The other component of the covariance estimate can be calculated using

$$
\mathrm{E}\left[\boldsymbol{\theta}(k) \boldsymbol{\theta}(k)^{T} \mid \boldsymbol{Z}(k)\right] \approx \sum_{j=1}^{n} \boldsymbol{\theta}_{j} \boldsymbol{\theta}_{j}^{T} w_{j}(\boldsymbol{Z}(k)) .
$$

\section{Motion Model}

This section describes a state space model for describing the motion of a mobile terminal located in a road vehicle. A state space model describes the time evolution of the location state of the mobile terminal in terms of differential equations in continuous time, or time difference equations in discrete time. The true location of a mobile terminal is described in three dimensions. In this paper we estimate the two dimensional location of the mobile terminal which is suitable for vehicle mobile terminal location. The work is easily extended to three dimensions.

A vehicle is subject to several friction and drag forces which oppose the acceleration of the vehicle as the vehicle's velocity increases. The result of this is that if the vehicle is subject to constant driving force, the acceleration of the vehicle will decrease as the velocity increases.

A kinematic model with a proportional negative feedback term to model the effects of air and rolling resistance is proposed. $\left[p_{x}(t), p_{y}(t)\right]^{T}$ is the location vector of the mobile terminal, and $\left[v_{x}(t), v_{y}(t)\right]^{T}$ is the velocity vector of the mobile terminal. A state vector is defined as $\boldsymbol{X}(t)=\left[p_{x}(t) v_{x}(t) p_{y}(t) v_{y}(t)\right]^{T}$. In continuous time, the state space model of vehicular motion can be given by

$$
\begin{aligned}
\dot{\boldsymbol{X}}(t)=\boldsymbol{A} \boldsymbol{X}(t)+\boldsymbol{B}\{\boldsymbol{W}(t)+\boldsymbol{U}(t)\} & {\left[\begin{array}{cccc}
0 & 1 & 0 & 0 \\
0 & -\alpha & 0 & 0 \\
0 & 0 & 0 & 1 \\
0 & 0 & 0 & -\alpha
\end{array}\right] \boldsymbol{X}(t)+} \\
& {\left[\begin{array}{ll}
0 & 0 \\
1 & 0 \\
0 & 0 \\
0 & 1
\end{array}\right]\left\{\left[\begin{array}{l}
\boldsymbol{W}_{x}(t) \\
\boldsymbol{W}_{y}(t)
\end{array}\right]+\left[\begin{array}{l}
\boldsymbol{U}_{x}(t) \\
\boldsymbol{U}_{y}(t)
\end{array}\right]\right\} . }
\end{aligned}
$$

The terms $\boldsymbol{W}_{x}(t)$ and $\boldsymbol{W}_{y}(t)$ represent zero mean white Gaussian noise processes with variances of $\mathrm{E}\left[\boldsymbol{W}_{x}(0)^{2}\right]=$ $\mathrm{E}\left[\boldsymbol{W}_{y}(0)^{2}\right]=\sigma^{2}$ which are the process noise terms for the continuous time dynamic model. The process noise models random effects such as noise in the control system of the vehicle, variations between drivers, and random road conditions. The deterministic inputs, representing driver control input in the $x$ and $y$ directions are given by $\boldsymbol{U}_{x}(t)$ and $\boldsymbol{U}_{y}(t)$. The control inputs are the drivers input into the system which controls the direction the vehicle is moving, in which direction it will accelerate, and so on. The constant $\alpha$ is a drag coefficient that models the various friction and resistance forces acting on the vehicle. If the control inputs change, the mobile terminal motion will smoothly change to the new direction of motion as the drag term forces the velocity functions to remain continuous. For maneuvering vehicles in North American urban environments a value of $\alpha=\frac{1}{6}$, $\sigma^{2}=\frac{1}{3}$ (meters/second $\left.^{2}\right)^{2}$, and $|\boldsymbol{U}(t)|=2.5$ meters/second ${ }^{2}$ gives a good match to observed vehicular behavior for straight line acceleration[7].

In practice, we can only sample measurements of the state of the system at discrete times. We will assume that the state is 
sampled with a sampling period of $T$ seconds. The discrete state vector is given by $\boldsymbol{X}(k)=\left[p_{x}(k T) v_{x}(k T) p_{y}(k T) v_{y}(k T)\right]^{T}$.

In field implementations, a location estimator works with measurements sampled at discrete times. A discrete time model for mobile terminal motion is thus required. The discrete version of the dynamic model can be obtained from the continuous time model[8]. We make the simplifying assumption that the input vector $\boldsymbol{U}(t)$ changes only at the sample times. Obviously, the inputs can change at any time instant not just at the sampling instants. The error introduced by this mismatch between the modeling assumptions and real model will be negligible provided the sampling period is small compared to the time constant of the continuous system, $\alpha^{-1}$. The sampling period is set at $T=0.5$ seconds which is less than the time constant of the system of $1 / \alpha=6.0$ seconds which justifies the assumption made to discretize the continuous state space model. The resulting discrete time dynamic model is given by

$$
\boldsymbol{X}(k+1)=\boldsymbol{\Phi} \boldsymbol{X}(k)+\Gamma \boldsymbol{U}(k)+\boldsymbol{W}(k),
$$

where

$$
\begin{gathered}
\boldsymbol{\Phi}=\left[\begin{array}{cccc}
1 & \frac{(1-\exp (-\alpha T))}{\alpha} & 0 & 0 \\
0 & \exp (-\alpha T) & 0 & 0 \\
0 & 0 & 1 & \frac{(1-\exp (-\alpha T))}{\alpha} \\
0 & 0 & 0 & \exp (-\alpha T)
\end{array}\right], \\
\boldsymbol{\Gamma}=\left[\begin{array}{cc}
\frac{\exp (-\alpha T)-1+\alpha T}{\alpha^{2}} & 0 \\
\frac{1-\exp (-\alpha T)}{\alpha} & 0 \\
0 & \frac{\exp (-\alpha T)-1+\alpha T}{\alpha^{2}} \\
0 & \frac{1-\exp (-\alpha T)}{\alpha}
\end{array}\right],
\end{gathered}
$$

and

$$
\boldsymbol{Q}=E\left[\boldsymbol{W}(k) \boldsymbol{W}(k)^{T}\right]=\left[\begin{array}{cccc}
r_{11} & r_{12} & 0 & 0 \\
r_{12} & r_{22} & 0 & 0 \\
0 & 0 & r_{11} & r_{12} \\
0 & 0 & r_{12} & r_{22}
\end{array}\right]
$$

The components of the process noise covariance $Q$ are given by

$$
\begin{aligned}
& r_{11}=\frac{\sigma^{2}(2 \alpha T-3+4 \exp (-\alpha T)-\exp (-2 \alpha T))}{2 \alpha^{3}}, \\
& r_{12}=\frac{\sigma^{2}(1-\exp (-\alpha T))^{2}}{2 \alpha^{2}}, \text { and } \\
& r_{22}=\frac{\sigma^{2}(1-\exp (-2 \alpha T))}{2 \alpha} .
\end{aligned}
$$

For hand off measurements, mobile terminals make measurements of the signal for the base stations they are using for primary communications but also of the signal from other base stations. It is likely to be these measurements that will be extended for mobile terminal location purposes. Therefore, the sampling period was set to the approximate the time between measurements in support of the hand off algorithm in GSM. Other networks standards, e.g. IS-95, have different sampling intervals for hand off but the hand off sampling periods are of the same order of magnitude so the results are still valid.

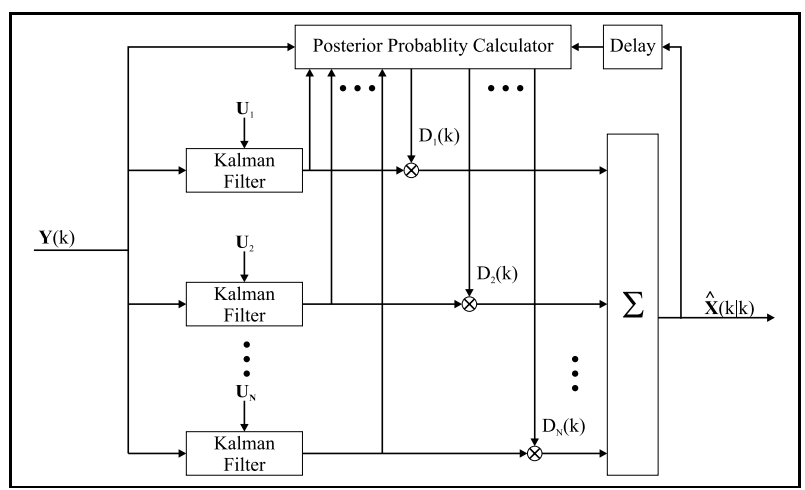

Fig. 2. Interactive Multiple Model Filter Structure

The control input, $\boldsymbol{U}(k)$, is usually equal to a member of a discrete set of vectors each matching motion along the direction of the streets, $\boldsymbol{U}(k) \in\left\{\boldsymbol{U}_{1}, \boldsymbol{U}_{2}, \ldots, \boldsymbol{U}_{N}\right\}$. For a Manhattan street layout such as shown in Figure 3, $N=5$ with one control input for each of the cardinal directions plus one additional vector for an all zero control input vector. The control input in most cases changes only when the mobile terminal is located in an intersection, otherwise $\boldsymbol{U}(k)=\boldsymbol{U}(k-1)$.

The performance of a Kalman filter based on the above dynamic model is compared with a Simple Kalman filter based on the following dynamic model from [3]. For this filter:

$$
\boldsymbol{\Phi}=\left[\begin{array}{llll}
1 & T & 0 & 0 \\
0 & 1 & 0 & 0 \\
0 & 0 & 1 & T \\
0 & 0 & 0 & 1
\end{array}\right] \text { and } \boldsymbol{Q}=\left[\begin{array}{cccc}
0 & 0 & 0 & 0 \\
0 & c & 0 & 0 \\
0 & 0 & 0 & 0 \\
0 & 0 & 0 & c
\end{array}\right],
$$

where $c$ is a value optimized for minimum expected squared error. This model does not include the effects control input so $\Gamma$, for this model, is a matrix of all zeros.

\section{Filtering and State Estimation}

The Zero Memory Estimator provides the filter with an estimate of mobile terminal position, $\boldsymbol{Y}(k)$. This estimated position is a linear function of the mobile terminal state $\boldsymbol{X}(k)$ :

$$
\boldsymbol{Y}(k)=\boldsymbol{H} \boldsymbol{X}(k)+\boldsymbol{V}(k),
$$

where $\boldsymbol{H}=\left[\begin{array}{llll}1 & 0 & 0 & 0 \\ 0 & 0 & 1 & 0\end{array}\right]$ and $\boldsymbol{V}(k)$ is the error of the zero memory estimator. The covariance of $\boldsymbol{V}(k), \boldsymbol{R}(k)$, is estimated by the Zero Memory estimator as described in Section II-A.

If the control input vector, $\boldsymbol{U}(k)$, is known, the optimal estimation algorithm for $\boldsymbol{X}(k)$ is the well known Kalman Filter[9]. Unfortunately, the control input must be estimated in parallel with the mobile terminal location and velocity.

A Bayesian estimator that makes use of the discrete nature of the control inputs is proposed in this paper. The location system state of the mobile terminal is estimated using a multi-model filter as shown in Figure 2. Multiple Kalman filters, each matched to one of the possible control inputs, are run in parallel. These 


\begin{tabular}{ccc}
\multicolumn{3}{c}{$\operatorname{Pr}\left(\boldsymbol{U}(k+1)=\boldsymbol{U}_{i} \mid \boldsymbol{U}(k)=\boldsymbol{U}_{j}, \boldsymbol{X}(k)=\hat{\boldsymbol{X}}(k \mid k)\right.$} \\
\hline$\hat{\boldsymbol{X}}(k \mid k)$ & $i=j$ & $i \neq j$ \\
\hline Not in Intersection & 0.9999 & $\frac{1-0.9999}{4}$ \\
\hline In Intersection & $\operatorname{Pr}($ TOSELF $)$ & $\frac{1-\operatorname{Pr}(\text { TOSELF })}{4}$ \\
\hline
\end{tabular}

TABLE I

TRANSITION PROBABILITIES

filters interact as the control input can switch from one possible input vector to another. The final state estimate for time interval $k$ is given by $\hat{\boldsymbol{X}}(k \mid k)=\sum_{j=1}^{N} \boldsymbol{D}_{j}(k) \boldsymbol{X}_{j}(k \mid k)$, where $\boldsymbol{X}_{j}(k \mid k)$ is the output of the $j^{\text {th }}$ Kalman filter and $\boldsymbol{D}_{j}(k)$ is the weight vector defined as the probability of model $j$ being the true model given the observed measurements[10]. The probabilities are calculated using

$$
\begin{array}{r}
\boldsymbol{D}_{i}(k+1)= \\
\frac{f\left(\boldsymbol{Y}(k+1) \mid \boldsymbol{U}(k)=\boldsymbol{U}_{i}, \boldsymbol{Y}(k), \boldsymbol{Y}(k-1), \ldots, \boldsymbol{Y}(1)\right)}{f(\boldsymbol{Y}(k+1) \mid \boldsymbol{Y}(k), \boldsymbol{Y}(k-1), \ldots, \boldsymbol{Y}(1))} \\
\sum_{j=1}^{N} \hat{\boldsymbol{\Theta}}_{i j}(k) \boldsymbol{D}_{j}(k),
\end{array}
$$

where $\hat{\boldsymbol{\Theta}}(k)$ is a matrix specifying the transition probabilities between the different control inputs given the current estimated location of the terminal, $\hat{\boldsymbol{\Theta}}_{i j}(k)=\operatorname{Pr}\left[\boldsymbol{U}(k+1)=\boldsymbol{U}_{i} \mid \boldsymbol{U}(k)=\right.$ $\left.\boldsymbol{U}_{j}, \boldsymbol{X}(k)=\hat{\boldsymbol{X}}(k \mid k)\right]$. The density value in the numerator can be calculated using outputs from the Kalman filter and zero memory estimator. The value of $\boldsymbol{Y}(k+1)$ given all past measurement values assuming that the control input $\boldsymbol{U}(k)=\boldsymbol{U}_{i}$ is Gaussian with mean $\boldsymbol{X}_{i}(k+1 \mid k)=\boldsymbol{\Phi} \boldsymbol{X}_{i}(k \mid k)+\boldsymbol{\Gamma} \boldsymbol{U}_{i}$, and variance $\boldsymbol{H} \boldsymbol{P}_{i}(k+1 \mid k) \boldsymbol{H}^{T}+\boldsymbol{R}(k)$ where $\boldsymbol{X}_{i}(k \mid k)$ is the output of the $i^{t h}$ Kalman filter for sample interval $k$, and $\boldsymbol{P}_{i}(k+1 \mid k)$ is the variance of $\boldsymbol{X}_{i}(k+1 \mid k)[10]$. The denominator value in (11) is a constant for all the filters and does not need to be computed.

An innovation introduced in this paper is using the the fact that the transition probabilities are a function of location to improve the accuracy of the estimation algorithm. The control input transition probabilities are calculated using knowledge of the environment. At intersections, the control input is likely to change. When the mobile terminal is between intersections, the probability is high that the control input will remain the same. The filtering algorithm incorporates this knowledge into the estimation algorithm. The transition probabilities used in this paper are given in Table I where $\operatorname{Pr}(T O S E L F)$ is a user selected constant proportional to the probability the user will turn at each intersection.

If the input vector changes after the control input has remained unchanged for several sample intervals, it takes the Kalman filter which is matched to the new input several sampling intervals to converge to the system state of mobile terminal. The newly matched Kalman filter must first remove the errors from its location estimate. Meanwhile, the Kalman filter which matches the previous input vector will generate state estimates with asymptotically increasing errors as the effects of the new mismatch build up. The effect of these transition effects is that the control input estimation algorithm requires several sampling periods to properly identify the new input vector.

One method to increase the rate at which the Kalman Filters adjust to input vector changes is to elevate the assumed covariance of the process noise over the value given in the mobility model[10]. This increased covariance reflects uncertainty in the knowledge of the input vectors. The process noise covariance, a design parameter in the setting of Kalman filters, is set to

$$
\boldsymbol{Q}=\boldsymbol{Q}_{\text {model }}+\boldsymbol{\Gamma} Q_{u} \boldsymbol{I} \boldsymbol{\Gamma}^{T},
$$

where $\boldsymbol{Q}_{\text {model }}$ is the process noise in the Kinematic model of the mobility model, $\boldsymbol{I}$ is an appropriately sized identity matrix, and $Q_{u}$ is a positive scalar constant.

The optimal value is $Q_{u}$ is a trade off between the performance of the Kalman filter with the correct input vector, and the performance of the filters with mismatched input vectors. Low values of $Q_{u}$ will give asymptotically better performance if the input vector remains constant for a long period of time at the cost of longer convergence times after the input vector changes. The range of values for $Q_{u}$ which give reasonable estimator performance is from 0 to the variance of the control input in one of the coordinate axis directions. In this application, the control input for either the $x$ or $y$ directions can go from -2.5 to 2.5 where 2.5 meters/second ${ }^{2}$ is the maximum acceleration of the mobile. Since the mean of the control inputs is 0 , it can be easily shown that the variance of the control input along one axis in this application is less than $2.5^{2}$, therefore $0 \leq Q_{u}<6.25$.

\section{RESUlTS}

The estimation method is applied to a mobile terminal moving through a simulated dense urban environment shown in Figure 3 with block lengths of 300 meters and street widths of 20 meters. As the terminal moves, it encounters Line of Sight and Non Line of Sight propagation. A realistic turning behavior model is used where the mobile terminal slows down before making a turn at an intersection. At each sampling interval, ToA measurements from the closest base stations and the two other base stations with the lowest distance measurements are used by the Zero Memory estimation algorithm to calculate the input to the filtering algorithm.

For the first set of simulations, the mobile has a $\frac{2}{3}$ probability of turning when it encounters an intersection. We compare our filter results with those obtained using the filter in [3]. The filter parameters were set to $\operatorname{Pr}(T O S E L F)=0.80$ and $Q_{u}=3.15$, the values that were empirically found to give the lowest errors for $\operatorname{Pr}(T U R N)=\frac{2}{3}$. The parameters of the simple Kalman Filter were also optimized to $c=1.5$, the optimal value also found empirically. It was also tried to calculate the value of $c$ for the simple Kalman Filter using the method described in [3], the resulting error curve labeled as 'Simple Kalman Filter(Hellebrandt).'. This optimization method does not work well as it does not give sufficient weight to the rapid maneuvers in 


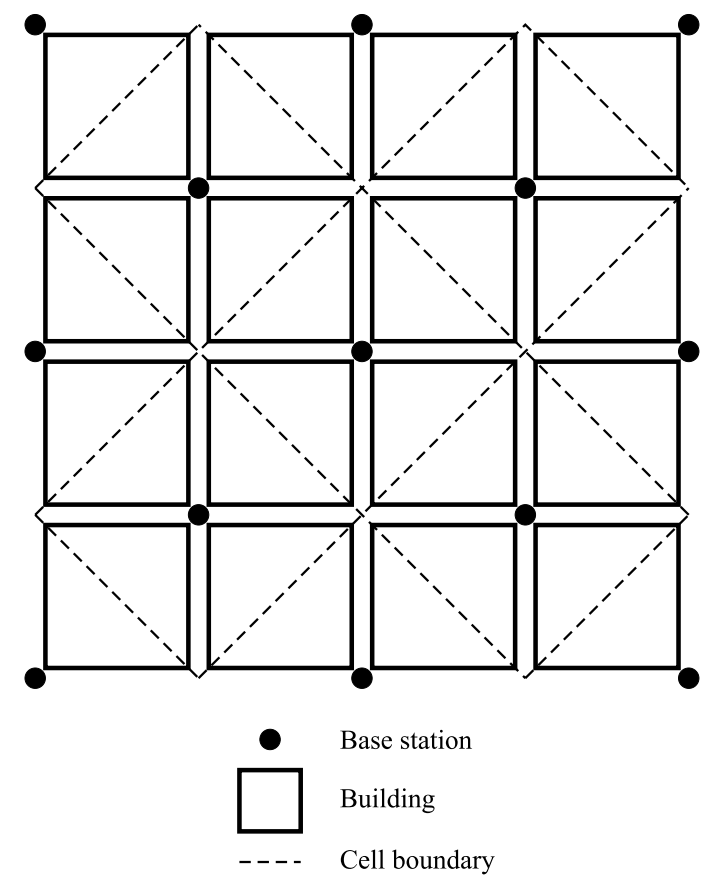

Fig. 3. Manhattan street layout

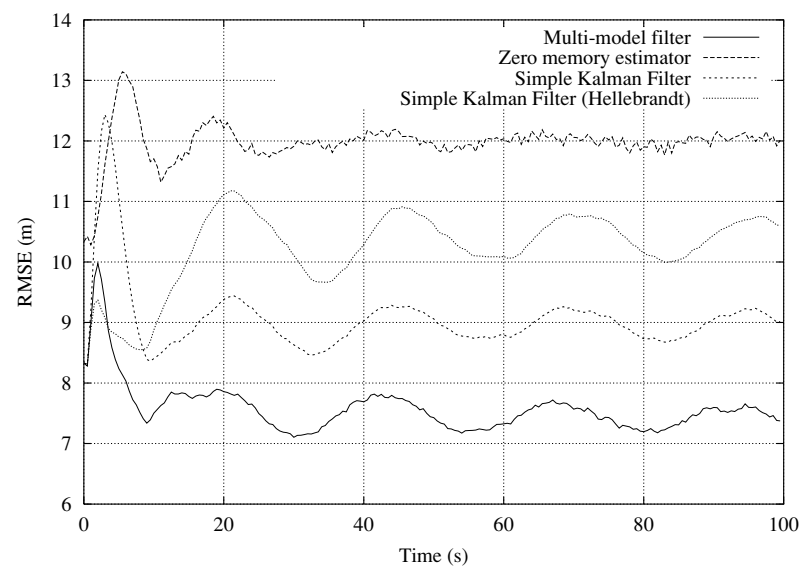

Fig. 4. Filtering Location Accuracy Results

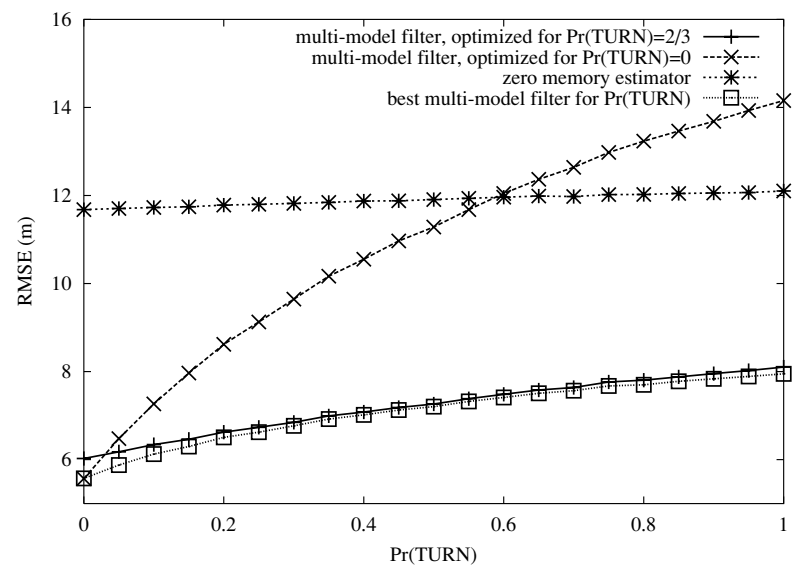

Fig. 5. Filtering Location Robustness Results intersections. Figure 4 shows that the new filter provides better location accuracy with less transient behavior when tracking is started.

The second set of simulations demonstrates the robustness of the new filtering algorithm. The probability of the mobile turning at each intersection was varied and the mean squared location error averaged over the first 100 seconds of the mobile terminal run is reported. The results for the multi-model filter optimized for different turning probabilities are compared with using only the Zero Memory Estimator. It can be seen that using a filter optimized for a turning probability of $\frac{2}{3}$ is robust to a wide range of true turning probabilities.

\section{CONCLUSIONS}

This paper presents a dynamic filter for the mobile terminal location problem. This filter is based upon a realistic motion model based on observations of vehicular motion. A zero memory estimator preprocesses the raw measurement data creating a pseudo-measurement that is linear with respect to the terminal location vector. A multi-model algorithm is then used to filter the mobile terminal location estimates. This filter uses the knowledge that the control inputs transition probabilities are a function of mobile terminal location to improve estimation accuracy. It is demonstrated that the new filter provides better accuracy than previously presented filters and is robust to changes in the motion parameters.

\section{REFERENCES}

[1] J.J. Caffery, Jr and G.L. Stüber, "Overview of radiolocation in CDMA cellular systems," IEEE Communications Magazine, vol. 36, no. 4, pp. 38-45, April 1998.

[2] M.A. Spirito, S. Pöykkö, and O. Knuuttila, "Experimental performance of methods to estimate the location of legacy handsets in GSM," in Fall Vehicular Technology Conference, October 2001, pp. 2716-2720.

[3] M. Hellebrandt and R. Mathar, "Location tracking of mobiles in cellular radio networks," IEEE Transactions on Vehicular Technology, vol. 48, no. 5, pp. 1558-1562, September 1999.

[4] P. Bernadin, M.F. Yee, and T. Ellis, "Cell radius inaccuracy: A new measure of coverage reliability," IEEE Transactions on Vehicular Technology, vol. 47, no. 4, pp. 1215-1226, November 1998.

[5] R.R. Collman, "Evaluation of methods for determining the mobile traffic distribution in cellular radio networks," IEEE Transactions on Vehicular Technology, vol. 50, no. 6, pp. 1629-1635, November 2001.

[6] M. McGuire, K.N. Plataniotis, and A.N. Venetsanopoulos, "Location of mobile terminals using time measurements and survey points," Submitted to IEEE Transactions on Vehicular Technology, June 2001.

[7] W.D. Glauz and D.W Harwood, "Chapter 3: Vehicles," in Traffic Engineering Handbook, J.L. Pline, Ed. Institution of Transportation Engineers, Washington, D.C., 5th edition, 1999.

[8] K. Ogata, Discrete-Time Control Systems, Prentice Hall, Englewood Cliffs, N.J., 1995.

[9] E. Brookner, Tracking and Kalman Filtering Made Easy, John Wiley \& Sons, Inc., Toronto, Ontario, Canada, 1998.

[10] R.L. Moose, H.F. VanLandingham, and D.H. McCabe, "Modeling and estimation for tracking maneuvering targets," IEEE Transactions on Aerospace and Electronic Systems, vol. AES-15, no. 3, pp. 448-456, May 1979. 